\title{
CARACTERIZAÇÃO DA FARINHA DE BAGAÇO DE JABUTICABA E PROPRIEDADES FUNCIONAIS DOS EXTRUSADOS ${ }^{1}$
}

\author{
Diego Palmiro Ramirez ASCHERI ${ }^{2 *}$, José Luis Ramírez ASCHERI ${ }^{3}$, Carlos Wanderlei Piller de CARVALHO ${ }^{3}$
}

\begin{abstract}
RESUMO
Estudaram-se as características de farinha de bagaço de jabuticaba fermentado e algumas propriedades funcionais (índice de expansão e índice de solubilidade) dos extrusados elaborados a partir deste bagaço e arroz, em proporções que variaram de 11,59 a $25 \%$ (bagaço/arroz). A farinha de bagaço de jabuticaba fermentado foi avaliada quanto à distribuição do tamanho de partícula, cor, sabor e aroma, composição centesimal, minerais e fibras. Algumas das características desta farinha foram comparadas com as das farinhas de bagaço de jabuticaba doce (sem fermentar) e arroz polido. O extrusor utilizado para obtenção das farinhas pré-gelatinizadas constituiu-se em um único parafuso com taxa de compressão de 1:3, com rotação variável (65,90 a 234,10 rpm). Usou-se uma matriz circular de $3 \mathrm{~mm}$ e uma taxa de alimentação de $5 \mathrm{~kg} / \mathrm{h}$. As temperaturas da $1^{\text {a }}$ e $2^{\text {a }}$ zonas de aquecimento do extrusor foram mantidas fixas (50 e $100{ }^{\circ} \mathrm{C}$, respectivamente) e a $3^{\mathrm{a}}$ zona variou entre 99,54 e $200,46{ }^{\circ} \mathrm{C}$. A Metodologia de Superfície de Resposta foi usada para avaliar a tendência das propriedades funcionais frente às variações de bagaço na formulação e dos parâmetros do processo (temperatura e rotação do parafuso). Os resultados mostraram que a farinha de bagaço de jabuticaba fermentado é uma boa fonte de fibras, de proteínas e de minerais, constituída de partículas finas com aroma característico do fruto e de sabor azedo-adstringente e ligeiramente salgado. A expansão dos extrusados aumentou com o aumento da rotação do parafuso e diminuiu com o aumento da adição de farinha de bagaço de jabuticaba, apresentando um comportamento quadrático com o aumento da temperatura. O índice de solubilidade aumentou com o aumento da rotação do parafuso e com a diminuição da temperatura. Maiores índices de expansão (3,26) e de solubilidade $(17,55 \%)$ foram alcançados numa formulação contendo aproximadamente $10 \%$ de bagaço de jabuticaba fermentado a $240 \mathrm{rpm}$ e temperaturas de 130 e $100^{\circ} \mathrm{C}$, respectivamente. A farinha de bagaço de jabuticaba fermentado misturada com farinha de arroz polido poderá ser usada como matéria-prima para a produção de farinhas pré-gelatinizadas, se as circunstâncias operacionais apropriadas forem aplicadas.
\end{abstract}

Palavras-chave: extrusão termoplástica, farinha de arroz, jabuticaba, metodologia de superfície de resposta, aproveitamento de resíduo agroindustrial.

\section{SUMMARY}

CHARACTERIZATION OF JABOTICABA BAGASSE FLOUR AND FUNCTIONAL PROPERTIES OF EXTRUDATES. The physical properties of fermented Jaboticaba bagasse flour and some functional properties (expansion index and water solubility index) of the extrudates obtained from mixtures of rice and Jaboticaba bagasse flour were studied, which varied from 11.59 to $25.00 \%$ (bagasse/rice). The fermented Jaboticaba bagasse flour analyzed the particle size distribution, color, flavor and chemical composition. Some of these properties were compared with the non-fermented Jaboticaba bagasse and white rice. A single screw extruder with a compression rate of $1: 3$ fitted with a round die of $3 \mathrm{~mm}$ diameter was used at a variable screw speed (65.9 to $234.1 \mathrm{rpm})$ and feed rate at $5 \mathrm{~kg} / \mathrm{h}$. The temperature of the extruder heating zones 1 and 2 were kept constant at 50 and $100{ }^{\circ} \mathrm{C}$, respectively and zone 3 varied from 99.54 to $200.46{ }^{\circ} \mathrm{C}$. Surface response methodology was used to show the effect of bagasse concentration and the parameters (temperature and screw speed). The results showed that fermented jaboticaba bagasse is a good source of fiber, proteins and minerals and is made up of fine particles with the aroma of the fruit and slight salty bitter taste. Radial expansion increased as the screw speed increased and decreased as the jaboticaba bagasse flour increased showing a quadratic effect as the temperature increased. Water solubility index increased as the screw speed increased and reduced as the temperature decreased. High expansion index (3.26) and water solubility (17.55\%) were reached for the formulation with $10 \%$ fermented jaboticaba bagasse at $240 \mathrm{rpm}$ screw speed and at 130 and $100{ }^{\circ} \mathrm{C}$ temperatures, respectively. Fermented jaboticaba bagasse flour can be incorporated into rice flour to produce pre-gelatinized flour if appropriate operational conditions are used.

Keywords: thermoplastic extrusion, rice flour, jabuticaba, response surface methodology, agroindustry by-products.

\section{1 - INTRODUÇÃO}

A jabuticabeira é uma planta nativa do Brasil, pertence à família Myrtaceae, podendo ser encontrada desde o Estado

\footnotetext{
${ }^{1}$ Recebido para publicação em 27/3/2006. Aceito para publicação em 20/10/2006 (001709)

${ }^{2}$ Unidade Universitária de Ciências Exatas e Tecnológicas,

Universidade Estadual de Goiás (UEG), Br 153, Km 3, C. P. 459,

Bairro Arco Verde, CEP 75110-390, Anápolis (GO), Brasil,

E-mail: ascheridpr@uol.com.br

${ }^{3}$ Embrapa Agroindústria de Alimentos, Av. das Américas, 29501,

Guaratiba, CEP 23020-470, Rio de Janeiro (RJ), Brasil,

E-mail:cwpiler@ctaa.embrapa.br; ascheri@ctaa.embrapa.br.

* A quem a correspondência deve ser enviada
}

do Pará até o do Rio Grande do Sul, mas é nos Estados de São Paulo, Rio de Janeiro, Minas Gerais e Espírito Santo que ocorrem as maiores produções. Dentre as espécies atualmente conhecidas, destacam-se a Myrciaria cauliflora (DC) Berg (jabuticaba paulista ou jabuticaba açu) e a Myrciaria jabuticaba (Vell) Berg (jabuticaba sabará), que produzem frutos apropriados tanto para a indústria como para consumo in natura [17, 38].

Segundo WILBANK et al. [47], o fruto de jabuticaba é uma baga, subgloboso, negro, quando maduro, liso, com 1,6 a 2,2 cm de diâmetro, contendo de 1 a 4 sementes. A casca é fina e muito frágil; a polpa é doce com leve acidez, de ótimo sabor e de cor branca a translúcida. 
De acordo com ASQUIERI et al. [5] e BRUNINI et al. [12], a jabuticaba é altamente perecível apresentando um período curto de comercialização após a colheita [6] devido ao elevado teor de água e açúcares e outros constituintes presentes na polpa. Em frutos de jabuticaba "sabará" cultivadas na Região Centro-Oeste do Brasil, ASQUIERI et al. [5] obtiveram teores de umidade, lipídios e proteínas de 83,3, 2,1 e $1,0 \mathrm{~g}$ em $100 \mathrm{~g}$ de polpa de jabuticaba, respectivamente, e teores de açúcares totais, redutores e não redutores de 11,2, 9,3 e 1,9 g, respectivamente. Entretanto, OLIVEIRA et al. [41] pesquisaram a qualidade de jabuticabas "sabará" provenientes de 10 diferentes regiões de cultivos localizadas no Estado de São Paulo e detectaram a acidez titulável dos frutos entre 0,888 a 1,652 g de ácido cítrico por $100 \mathrm{~g}$ de polpa e o pH foi da ordem de 2,91 a 3,72. Os sólidos solúveis totais variaram entre 11,5 e $17,9{ }^{\circ} \mathrm{Brix}$; a vitamina $\mathrm{C}$, em torno dos 19,0 g de ácido ascórbico por $100 \mathrm{~g}$ de polpa; e os teores de potássio, magnésio e cálcio, entre 0,1 e 1,06 g, 0,07 e 0,6 g e entre 0,02 e 1,11 g por $100 \mathrm{~g}$ de polpa, respectivamente. BRUNINI et al. [12], em frutos desta mesma espécie vegetal, nas condições paulistas, determinaram teor de vitaminas em torno de 13,3 g de ácido ascórbico por 100 g de polpa e carboidratos solúveis de 2,66 g de glicose por $100 \mathrm{~g}$ de polpa.

ASQUIERI et al. [5], com o intuito de minimizar perdas econômicas significativas, estudaram o processo de produção de diferentes tipos de bebidas alcoólicas que poderiam ser fabricados a partir do fruto de jabuticaba "sabará". Os autores conseguiram produzir "vinho" branco, utilizando a polpa, e "vinho" tinto, utilizando a casca do fruto. Os mesmos autores [6] caracterizaram físico-química e sensorialmente bebidas alcoólicas similares a vinho tinto seco e doce, fabricados com a fruta integral da jabuticaba e constataram que o "vinho" doce é a bebida que teve maior preferência por parte dos consumidores.

ASCHERI et al. [3] e ASCHERI et al. [4], com a finalidade de aproveitar o bagaço obtido da prensagem das massas "vínicas", da indústria de "vinhos" de jabuticaba, produziram farinhas pré-gelatinizadas misturando o bagaço à farinha de arroz polido usando um processo de extrusão termoplástica. Além de algumas propriedades físico-químicas, os autores estudaram o efeito da extrusão sobre as propriedades de pasta e de adsorção de água e concluíram que o produto extrusado pode ser considerado como boa fonte de fibra alimentar com características peculiares para sua utilização industrial.

Por seu alto teor em fibras, o bagaço de jabuticaba pode ser reaproveitado em ingredientes que possam substituir parte das calorias de alimentos ricos em carboidratos, além de influenciar em vários aspectos da digestão, absorção e metabolismo [9].

Além de fibra alimentar, o bagaço pode fornecer sais minerais e proteínas remanescentes do agente biológico utilizado para a fermentação do suco de jabuticaba. Para isto, há necessidade de caracterizar o resíduo a fim de conhecer suas propriedades que possam facilitar seu uso em produtos alimentares.
Concordando com ASCHERI et al. [3] e ASCHERI et al. [4], uma das alternativas da utilização do bagaço de jabuticaba pode ser na elaboração de farinhas pré-gelatinizadas obtidas por extrusão, destinadas ao preparo de pudins, purê pré-pronto, bolo, bolacha, macarrão, até mesmo bebidas isotônicas, etc. A extrusão facilita a inclusão de fibras no material amiláceo e melhora as características organolépticas e funcionais do produto extrusado.

O índice de expansão (IE) e índice de solubilidade em água (ISA) são propriedades mais importantes dos produtos expandidos por extrusão. Por meio do IE, pode-se, em primeira observação, predizer a severidade do processo de extrusão [32, 34]. Este índice pode variar consideravelmente de acordo com as circunstâncias do processo [42] e da composição do alimento, como a presença de fibra alimentar. Segundo Lue et al. [33], da incorporação de níveis elevados de fibras resulta um produto extrusado expandido liso, compacto e rijo, com textura indesejável, em conseqüência do baixo índice de expansão.

O ISA depende da quantidade de moléculas solúveis e também da intensidade e do tipo de reações que ocorrem durante a extrusão. Além da gelatinização do amido, que resulta na liberação da amilose e amilopectina de seus grânulos, poderá ocorrer também a dextrinização dos componentes do amido e outras reações que conduzem à formação de compostos de baixo peso molecular, que irão influir no ISA [27, 44].

O presente trabalho teve por objetivo estudar as características da farinha de bagaço de jabuticaba fermentado e algumas propriedades funcionais dos extrusados elaborados a partir de mistura desta farinha com farinha de arroz polido, em diferentes condições do processo de extrusão.

\section{2 - MATERIAL E MÉTODOS}

\section{1 - Matéria-prima}

O bagaço doce e o bagaço fermentado foram fornecidos pela Fazenda Jabuticabal do município de Nova Fátima - GO. O bagaço doce foi coletado após o esmagamento do fruto durante a fabricação de sucos de jabuticaba e o bagaço fermentado foi coletado logo após o esgotamento total do suco fermentado por meio de prensagem, ao final do processo de fermentação do mosto de jabuticaba.

Após a coleta, os bagaços de jabuticaba foram desidratados e moídos no Laboratório de Pesquisa da Universidade Estadual de Goiás (Anápolis). Os bagaços, em separado, foram uniformemente distribuídos em bandejas e levados à estufa com recirculação de ar forçado da marca Tecnal à temperatura de $60{ }^{\circ} \mathrm{C}$ até atingir $7,0 \%$ de umidade. Os bagaços desidratados foram moídos em moinho de bolas, obtendo-se as farinhas de bagaço de jabuticaba fermentado (FBJ) e de bagaço fresco (BF).

O arroz (Oryza sativa, L.) polido da marca Blue-Platina foi adquirido do comércio da cidade do Rio de Janeiro, em sacos de $5 \mathrm{~kg}$. O arroz com umidade inicial de 12,63\%, foi 
moído em moinho de rolo tipo Quadrumat Junior da marca BRABENDER, obtendo-se a farinha de arroz polido (FAP).

\section{2 - Preparo das amostras por extrusão e delineamento experimental}

FAP e FBJ foram condicionadas em sacos plásticos de polietileno à umidade de $16 \%$, de acordo com a quantidade de massa seca preestabelecida em delineamento experimental do tipo central composto rotacional de $2^{\text {a }}$ ordem em que se usou metodologia de superfície de resposta [10], mostrada na Tabela 1, obtendo-se, desta maneira, as farinhas mistas cruas. Ao todo foram realizados 17 ensaios, sendo três deles repetições no ponto central.

$\mathrm{O}$ teor de umidade inicial das farinhas mistas que variou de $10,70 \%$ a $11,65 \%$ foi determinado segundo a AOAC [7] em estufa da marca BRABENDER, tipo MA a $130{ }^{\circ} \mathrm{C}$ por uma $\mathrm{h}$. A quantidade de água (que variou de 51,2 a 63,1 mL/1000 g de amostra) adicionada às misturas foi calculada por meio da fórmula seguinte:

$Q_{a}=\frac{\left(U_{f}-U_{i}\right)}{100-U_{f}} \cdot m_{a}$

onde, $\mathrm{Q}_{\mathrm{a}}=$ quantidade de água a adicionar $(\mathrm{mL}), \mathrm{U}_{\mathrm{f}}=\%$ de umidade final da amostra estabelecida a $16 \%, \mathrm{U}_{\mathrm{i}}=\% \mathrm{de}$ umidade inicial da amostra, e $\mathrm{m}_{\mathrm{a}}=$ massa da amostra ( $\mathrm{g}$ ).

Depois da homogeneização as amostras foram acondicionadas em sacos plásticos e refrigeradas por 24 hs para atingir a distribuição e absorção mais uniforme da água.

TABELA 1 - Arranjo de fatores usados na extrusão das farinhas mistas cruas de arroz e bagaço de jabuticaba.

\begin{tabular}{lcrrrr}
\hline Fatores & \multicolumn{5}{c}{ Nível } \\
\cline { 2 - 6 } & $\mathbf{- 1 , 6 8 2}$ & $\mathbf{- 1}$ & $\mathbf{0}$ & $\mathbf{+ 1}$ & $\mathbf{+ 1 , 6 8 2}$ \\
\hline $\mathrm{RP}(\mathrm{rpm})$ & 65,90 & 100 & 150 & 200 & 234,10 \\
$\mathrm{~T}\left({ }^{\circ} \mathrm{C}\right)$ & 99,54 & 120 & 150 & 180 & 200,46 \\
FBJ $(\%)$ & 11,59 & 15 & 20 & 25 & 28,41
\end{tabular}

$\mathrm{X}_{1}=\mathrm{RP}$; rotação do parafuso $(\mathrm{rpm}) ; \mathrm{X}_{2}=\mathrm{T}$; temperatura da $3^{\mathrm{a}}$ zona de aquecimento $\left({ }^{\circ} \mathrm{C}\right)$; $\mathrm{X}_{3}=\mathrm{FBJ}$; farinha de bagaço fermentado $(\%)$

As farinhas mistas cruas foram extrusadas num extrusor da marca BRABENDER, modelo 20 DN, constituída por um único parafuso com taxa de compressão de 1:3, cuja rotação variou segundo o delineamento experimental estabelecido na Tabela 1. Foi utilizada uma matriz circular de $3 \mathrm{~mm}$ e a taxa de alimentação foi de $5 \mathrm{~kg} / \mathrm{h}$. As temperaturas de aquecimento mantidas fixas foram de $50{ }^{\circ} \mathrm{C}$ na $1^{\text {a }}$ zona e de $100{ }^{\circ} \mathrm{C}$ na $2^{\text {a }}$ zona do canhão do extrusor. A temperatura da $3^{\text {a }}$ zona $\left(99,54\right.$ a $\left.200,46{ }^{\circ} \mathrm{C}\right)$ variou conforme o estabelecido no delineamento experimental (Tabela 1). O material extrusado foi coletado depois que o processo atingiu o equilíbrio visual obtido na leitura do torque do extrusor (aproximadamente de 15 Newton-metros). A coleta foi manual, cortando o material extrusado em tamanho aproximado de $30 \mathrm{~cm}$ de comprimento, acondicionados em bandejas de alumínio e desidratados em estufa com circulação de ar marca FABBER à temperatura de $75{ }^{\circ} \mathrm{C}$ até atingir uma umidade de 3 a $7 \%$. Em seguida, as amostras foram moídas em moinho de disco marca PERTEN e de rolos BRABENDER Junior, obtendo-se, assim, as farinhas mistas extrusadas (pré-gelatinizadas, FME). Após moagem, as FME foram acondicionadas em sacos plásticos, devidamente etiquetadas e armazenadas em temperatura ambiente.

\section{3 - Propriedades físicas e sensoriais}

Realizou-se análise qualitativa da forma das partículas, da FBJ empregando lupa estereomicroscópica da marca Carl Zeiss, modelo STEMI 2000-C com ocular 20x, objetivo 3x e câmera digital da marca SONY, modelo DSC-S75. As partículas analisadas foram obtidas da farinha através de peneiras vibratórias Produtest de malha de metal com abertura de 0,5 e $0,125 \mathrm{~mm}$ e tempo de peneiração de $10 \mathrm{~min}$.

A análise da distribuição dos tamanhos das partículas foi feita na unidade de dispersão líquida de um equipamento Laser Particle Sizer Analysette 22, o qual utiliza o princípio da difração a laser, produzindo laser hélio-neon no comprimento de onda de $632 \mathrm{~nm}$. Utilizou-se álcool isopropílico como agente dispersor das frações granulométricas que foram mantidas sob agitação constante até alcançar 7\% de obscuridade do feixe de laser e, então, iniciada a leitura.

Para avaliar o sabor, a cor e o aroma da FBJ foram criadas tabelas de referência. O aroma foi avaliado de acordo com a seguinte escala: 5 = sem odores desagradáveis, $3=$ odor desagradável leve, mas perceptível, 1 = odor desagradável forte. Para avaliar a coloração, foi utilizada a escala: 5 = Negro, 3 = roxo, 1 = roxo-claro. Para avaliar o sabor, foi construída a seguinte escala: 5 = azedo, 4 = azedo-adstringente, 3 = azedo-adstringente e ligeiramente salgado, 2 = adstringente, 1 = salgado. A elaboração dessas tabelas de referências se assemelha à descrita por TOIVONEN \& DEELL [45]. As amostras foram avaliadas por 10 provadores treinados e as notas compuseram a média final.

\section{4 - Composição centesimal}

A composição centesimal de BF, FBJ e FAP foi determinada em duplicata. Para a determinação da umidade, amostras destas farinhas foram pesadas e desidratadas em estufa MARCONI MA 035 a $105{ }^{\circ} \mathrm{C}$ até a obtenção de massa constante (precisão de $0,1 \mathrm{mg}$ ) [3]. Na análise de extrato etéreo, a amostra previamente desidratada foi submetida à extração com éter etílico por cerca de $5 \mathrm{~h}$, passando em seguida por processo de evaporação. O resíduo resultante foi seco a $105^{\circ} \mathrm{C}$ até obtenção de massa constante (precisão de $0,1 \mathrm{mg}$ ) [7]. Na amostra dessecada e desengordurada, foram determinadas as quantidades de cinzas e de proteínas. A proteína bruta foi determinada pelo método Kjeldahl tradicional [7], com modificações, usando como catalisadores o $\mathrm{Na}_{2} \mathrm{SO}_{4}, \mathrm{CUSO}_{4}$ e Se, titulado com $\mathrm{H}_{2} \mathrm{SO}_{4} 0,1 \mathrm{~N}$. Como fator de cálculo, foi usado o valor de 6,25. A quantidade de cinzas foi determinada segundo o método da AOAC [7].

\section{5 - Fibra detergente neutro e detergente ácido}

A quantidade de fibra em detergente neutro (FDN) e em detergente ácido (FDA) da farinha de bagaço de jabuticaba 
fermentado foi determinada de acordo com a metodologia descrita por SOUZA et al. [44].

\section{6 - Composição de minerais}

As determinações de cálcio, alumínio, fósforo, magnésio manganês molibdênio, sódio, potássio, selênio, zinco, cobre, ferro e chumbo da farinha de bagaço de jabuticaba fermentada foram realizadas de acordo com os métodos da AOAC [7]. A quantificação dos minerais foi realizada em espectrofotômetro de emissão de plasma ICP Spectroflama, sendo a determinação realizada por espectrometria de emissão atômica, depois de uma digestão completa da amostra com ácido nítrico e ácido perclórico.

\section{7 - Propriedades funcionais dos extrusados}

O índice de expansão (IE) foi calculado por meio da relação entre o diâmetro do material extrusado $(\mathrm{mm})$ e o diâmetro da matriz do extrusor $(\mathrm{mm})$. O valor considerado foi obtido por meio da media aritmética de 10 determinações para cada tratamento, conforme a metodologia proposta por GUJSKA \& KHAN [26].

$$
\mathrm{IE}=\frac{\text { Diâmetro do extendido }(\mathrm{mm})}{\text { Diâmetro da matriz }(\mathrm{mm})}
$$

O índice de solubilidade em água (ISA) foi determinado conforme o método descrito por ANDERSON [2]. Para o cálculo, foi utilizada a seguinte equação:

$$
\operatorname{ISA}(\%)=\frac{\text { Peso do resíduo evaporado }(\mathrm{g})}{\text { Peso da amostra }(\mathrm{g})} \times 100
$$

\section{8 - Análise estatística dos resultados}

Aplicou-se a equação de segunda ordem como modelo matemático preditivo das variáveis resposta $\mathrm{Y}_{1}$ (IE) e $\mathrm{Y}_{2}$ (ISA), cuja equação geral foi [10]:

$$
\begin{aligned}
& Y=b_{0}+b_{1} X_{1}+b_{2} X_{2}+b_{3} X_{3}+b_{11} X_{1}^{2}+b_{22} X_{2}^{2}+ \\
& b_{33} X_{3}^{2}+b_{12} X_{1} X_{2}+b_{13} X_{1} X_{3}+b_{23} X_{2} X_{3}+\xi
\end{aligned}
$$

onde $\mathrm{Y}$ é a função resposta genérica, $\mathrm{X}$ é a variável codificada, obtida a partir da variável original, b representa os coeficientes estimados pelos métodos dos mínimos quadrados e $\xi$ é o erro experimental.

Os gráficos de superfície de resposta e os coeficientes do modelo matemático foram obtidos por meio do programa Statistica for Windows v. 5.1 da StatSoft (1984-1996).

\section{3 - RESULTADOS E DISCUSSÃO}

\section{1 - Propriedades físicas e sensoriais}

O bagaço fresco desidratado caracteriza-se por ter cor roxo-escura intensa característica da fruta madura [39, 41], preservando parte da cor branca do endocarpo e da cor vermelha das sementes. O bagaço fermentado apenas difere do fresco pela cor vermelha do endocarpo, em decorrência da liberação dos pigmentos, e pela pele fina do exocarpo, pelo desprendimento do mesocarpo durante a fermentação.

De acordo com a avaliação sensorial quanto a cor, aroma e sabor da FBJ (Tabela 2), os 10 provadores coincidiram na cor e oito no aroma e no sabor e qualificaram a FBJ como sendo uma farinha de uma cor roxo-clara com um aroma sem odor desagradável (aroma característico do fruto) e com um sabor azedo-adstringente e ligeiramente salgado.

TABELA 2 - Avaliação da cor, aroma e sabor da farinha de bagaço de jabuticaba fermentada*.

\begin{tabular}{cccccc}
\hline \multicolumn{2}{c}{ Cor } & \multicolumn{2}{c}{ Aroma } & \multicolumn{2}{c}{ Sabor } \\
Escala & Nota & Escala & Nota & Escala & Nota \\
\hline 1 & 10 & 1 & 1 & 1 & 0 \\
- & - & - & - & 2 & 0 \\
3 & 0 & 3 & 1 & 3 & 8 \\
- & - & - & - & 4 & 2 \\
5 & 0 & 5 & 8 & 5 & 0 \\
\hline
\end{tabular}

*Média de três repetições.

Segundo o histograma da distribuição da freqüência dos tamanhos de partículas (Figura 1a), a FBJ apresentou aproximadamente $36 \%$ das partículas maiores que $0,5 \mathrm{~mm}$. Esta fração apresentou pedaços quebrados da semente e do exocarpo (Figura 1 b), cujas formas predominantes foram poliedros irregulares cúbicos e pentagonais, respectivamente. Mais de $64 \%$ das partículas apresentaram tamanhos menores que $0,5 \mathrm{~mm}$, caracterizando a FBJ como um produto desidratado em pó. Esta fração apresentou, predominantemente, fibras avermelhadas no mesocarpo e no endocarpo e fragmentos do exocarpo que adquirem forma de escamas de cor negra (Figura 1c).

\section{2 - Composição centesimal}

A farinha de bagaço de jabuticaba contém aproximadamente três vezes os teores de cinza e de proteínas do bagaço fresco, porém, contém duas vezes menos teor de extrato etéreo deste (Tabela 3). A diminuição do teor em extrato etéreo provavelmente se deve à diminuição de taninos, pigmentos e outras substâncias que migram para a água de maceração durante o processo de fermentação. A diferença entre a porcentagem de cinza e de proteína entre estas amostras se deve à adição e à proliferação de leveduras durante a fermentação do mosto de jabuticaba. Segundo ASQUIERI et al. [5], neste processo, adicionam-se aproximadamente $10 \mathrm{~g}$ de levedura de cerveja/L de mosto preparado, o que explica o aumento de proteínas e de resíduos minerais no bagaço fermentado.

Pela Tabela 3 se observa que a quantidade de proteína na FAP apenas difere da FBJ em 1,19 g/100 g de amostra seca, entretanto, se observa também que é pobre em cinzas, em extrato etéreo e em fibras.

\section{3 - Fibra detergente neutro e detergente ácido}

A determinação de fibras em detergente ácido e em detergente neutro mostrou que a FBJ é uma farinha rica 


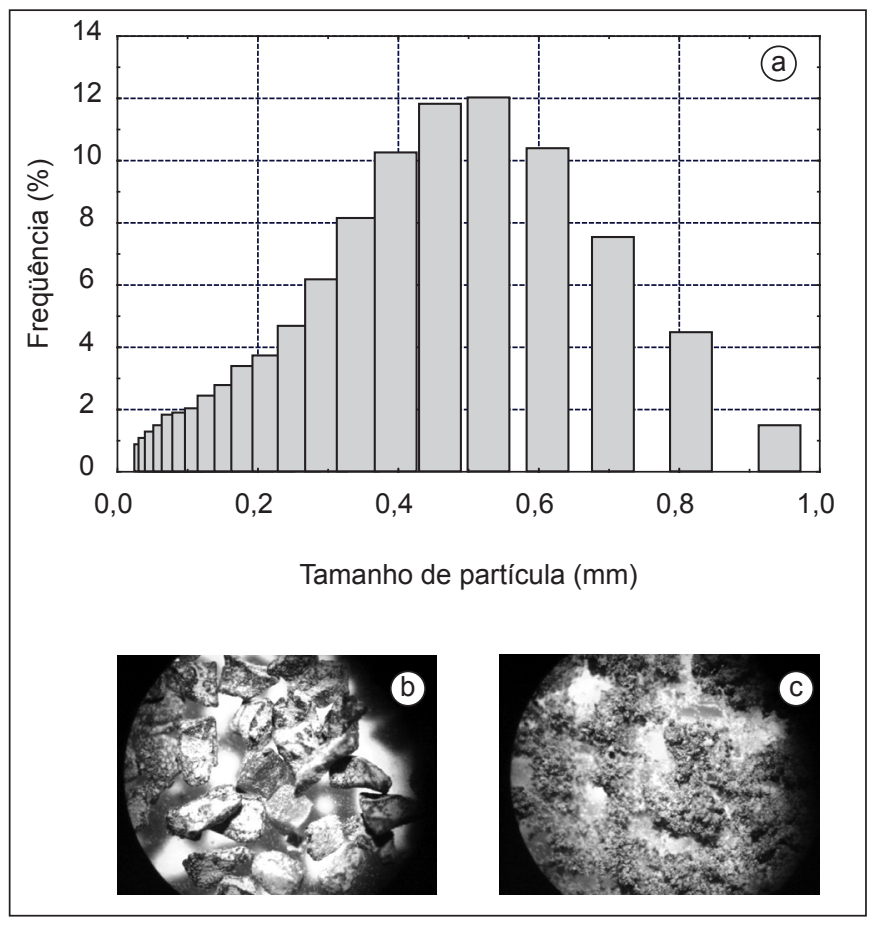

FIGURA 1 - Características físicas da farinha de bagaço de jabuticaba. a) Histograma da distribuição do tamanho de partícula, b) fragmentos de tamanho de partícula maiores de $0,5 \mathrm{~mm}$ e c) fragmentos de tamanho de partícula menores de $0,5 \mathrm{~mm}$.

TABELA 3 - Composição centesimal e de minerais das farinhas de bagaço de jabuticaba e de arroz polido.

\begin{tabular}{|c|c|c|c|}
\hline Parâmetro & BF* $^{*}$ & FBJ* & FAP* $^{*}$ \\
\hline \multicolumn{4}{|c|}{ Composição centesimal (em g/100 g) } \\
\hline Umidade & 7,32 & 7,08 & 12,01 \\
\hline Cinzas & 1,35 & 3,49 & 0,78 \\
\hline Extrato etéreo & 2,29 & 1,14 & 0,62 \\
\hline Proteína & 4,17 & 11,00 & 9,81 \\
\hline \multicolumn{4}{|c|}{ Fibra (g/100 g de amostra) } \\
\hline FDA & -- & 21,91 & -- \\
\hline FDN & -- & 45,47 & -- \\
\hline \multicolumn{4}{|c|}{ Composição em minerais $(\mathrm{mg} / 100 \mathrm{~g})$} \\
\hline Cálcio & -- & 114,28 & 6,49 \\
\hline Fósforo & -- & 195,29 & 77,48 \\
\hline Ferro & -- & 227,23 & 4,26 \\
\hline Alumínio & -- & 2,00 & -- \\
\hline Magnésio & -- & 124,50 & 54,62 \\
\hline Manganês & -- & 3,36 & 0,81 \\
\hline Potássio & -- & 1273,12 & 97,22 \\
\hline Zinco & -- & 6,80 & 2,01 \\
\hline Cobre & -- & 1,65 & -- \\
\hline Chumbo & -- & 0,18 & -- \\
\hline
\end{tabular}

*Média de duas repetições; e -- não determinado.

em fibras insolúveis apresentando valores de FDA e FDN de 21,91 e 45,47 g/100 g, respectivamente. De acordo com DREHER [18] um alimento com 2 a $3 \%$ de fibras pode ser considerado uma boa fonte de fibra alimentar. No Brasil, por meio da Resolução Normativa $n^{\circ} 27$ da Secretaria Nacional de Vigilância Sanitária [11], se estabelece, no regulamento técnico referente à informação nutricional complementar, que um alimento pode ser considerado fonte de fibra ali- mentar quando no produto acabado existirem $3 \mathrm{~g} / 100 \mathrm{~g}$ de fibras para alimentos sólidos e 1,5 g/100 mL de fibras para líquidos, já com o dobro deste conteúdo é considerado um alimento com elevado conteúdo de fibra alimentar.

\section{4 - Composição de minerais}

A Tabela 3 também mostra a composição em minerais da FBJ e da FAP. Quantidades mais elevadas foram observadas na FBJ. Os minerais $\mathrm{Ca}, \mathrm{P}, \mathrm{Fe}, \mathrm{Mg}, \mathrm{Mn}, \mathrm{K}$ e $\mathrm{Zn}$ presentes nesta farinha corresponderam a 18, 3, 53, 2, 4, 13 e 3 vezes a porcentagem dos respectivos minerais verificados na FAP. Entre estes minerais se destacam o ferro, o manganês e o cobre, fornecendo mais dos $100 \%$ da recomendação diária (IDR) na dieta de crianças e adultos [48].

Os valores de cálcio, fósforo e os demais elementos analisados na FBJ colocam este produto em evidência quando comparado à FAP. De acordo com a IDR destes elementos, a quantidade de FBJ utilizada para subministrar estes elementos seria menos de 68\%. Desta forma, a inclusão do bagaço de jabuticaba na dieta alimentar pode auxiliar no subministro destes importantes elementos para a saúde humana.

\section{5 - Propriedades funcionais dos extrusados}

A análise de variância (Tabela 4) mostrou que o ajuste do modelo proposto para determinar a variação dos índices de expansão e de solubilidade em água em função dos parâmetros de extrusão foi satisfatório. Os coeficientes de regressão das variáveis codificadas estão na Tabela 5, assim como também os coeficientes de correlação $\left(R^{2}\right)$ e respectivos valores do teste $t$ aplicado para sua significância estatística $(P<0,01)$.

TABELA 4 - Análise de variância referente ao modelo aplicado para índice de expansão (IE) e índice de solubilidade em água (ISA).

\begin{tabular}{clrrcc}
\hline Resposta & $\begin{array}{c}\text { Fonte de } \\
\text { variação }\end{array}$ & $\begin{array}{c}\text { Soma } \\
\text { quadrática }\end{array}$ & G. L. & $\begin{array}{c}\text { Média } \\
\text { quadrática }\end{array}$ & F \\
\hline IE & Regressão & 5,469 & 9 & 0,608 & $13,215^{* *}$ \\
& Resíduos & 1,747 & 38 & 0,046 & \\
& Falta de & & & & \\
& ajuste & 1,649 & 5 & 0,330 & $110,856^{* *}$ \\
& Erro puro & 0,098 & 33 & 0,003 & \\
& Total & 7,216 & 47 & & \\
\hline ISA & Regressão & 113,078 & 9 & 12,564 & $4,689^{*}$ \\
& Resíduos & 101,816 & 38 & 2,679 & \\
& Falta de & & & & \\
& ajuste & 95,429 & 5 & 19,086 & $98,618^{* *}$ \\
& Erro puro & 6,387 & 33 & 0,194 & \\
& Total & 214,894 & 47 & & \\
\hline
\end{tabular}

*Significância ao nível de $95 \%(P<0,05) ;{ }^{* *}$ Significância ao nível de $99 \%(P<0,01)$; e G. L. = graus de liberdade.

De acordo com o teste $t$ aplicado, os coeficientes de regressão significativos ao nível de 95 e 99\% foram considerados nos modelos matemáticos descritos nas equações de 5 a 8 . 
Usando as variáveis codificadas:

$Y_{1}=2,719+0,123 X_{1}-0,231 X_{2}-0,072 X_{3}-$

$0,244 X_{2}^{2}+0,036 X_{3}^{2}+0,028 X_{1} X_{2}$

$Y_{2}=13,275+0,957 X_{1}-1,244 X_{2}+0,456 X_{2}^{2}-0,213 X_{3}^{2}$

Usando as variáveis reais:

$\mathrm{IE}=-1,239+0,069 \mathrm{~T}-0,077 \mathrm{FBJ}-0,0003 \mathrm{~T}^{2}+$

$0,0014 \mathrm{FBJ}^{2}+0,00002 \mathrm{RPT}$

$I S A=17,314-0,048 R P+0,00018(R P)^{2}-0,00024 T^{2}$

Os termos lineares das variáveis codificadas (exceto $\mathrm{X}_{3}$ para $\mathrm{Y}_{2}$ ), seus termos quadráticos (exceto $\mathrm{X}_{2}^{1}$ e $\mathrm{X}_{3}^{2}$ para $\mathrm{Y}_{1} \mathrm{e}$ $\mathrm{Y}_{2}$, respectivamente) dos modelos $\mathrm{Y}_{1}$ e $\mathrm{Y}_{2}$ e a interação $\mathrm{X}_{12}$ do modelo $Y_{1}$ influenciaram nos valores experimentais das suas respectivas variáveis IE e ISA (Tabela 5). As mesmas observações foram feitas utilizando as variáveis reais com exceção da variável RP e a interação RP x T para IE e ISA, respectivamente, que não foram estatisticamente significativas $(P>0,05)$.

TABELA 5 - Teste $t$ e coeficientes estimados do modelo quadrático aplicado para as diferentes respostas estudadas.

\begin{tabular}{|c|c|c|c|c|}
\hline \multirow[t]{3}{*}{ Fator } & \multicolumn{4}{|c|}{ Coeficientes estimados } \\
\hline & \multicolumn{2}{|c|}{$Y_{1}$} & \multicolumn{2}{|c|}{$\mathbf{Y}_{2}$} \\
\hline & Coeficiente & Teste $t$ & Coeficiente & Teste $t$ \\
\hline $\mathrm{X}_{0}$ & $2,719^{* *}$ & 222,469 & $13,275^{* *}$ & 74,135 \\
\hline$X_{1}$ & 0,123 ** & 14,381 & $0,957^{* *}$ & 13,922 \\
\hline $\mathrm{X}_{2}$ & $-0,231 * *$ & $-27,063$ & $-1,244 * *$ & $-18,103$ \\
\hline$x_{3}$ & $-0,072 * *$ & $-8,482$ & $-0,061$ n.s. & $-0,882$ \\
\hline $\mathrm{X}_{1}^{2}$ & - 0,003 n.s. & $-0,272$ & $0,456 * *$ & 5,467 \\
\hline $\mathrm{X}_{2}^{2}$ & $-0,244 * *$ & $-23,617$ & $-0,213 * *$ & $-2,550$ \\
\hline$X_{3}^{2}$ & $0,036 * *$ & 3,477 & 0,021 n.s. & 0,251 \\
\hline $\mathrm{X}_{12}$ & 0,028 * & 2,510 & $-0,003$ n.s. & $-0,034$ \\
\hline $\mathrm{X}_{13}$ & $-0,005$ n.s. & $-0,421$ & 0,153 n.s. & 1,699 \\
\hline $\mathrm{X}_{23}$ & 0,008 n.s. & 0,733 & $-0,023$ n.s. & $-0,257$ \\
\hline$R^{2}$ & \multicolumn{2}{|c|}{0,526} & \multicolumn{2}{|c|}{0,989} \\
\hline
\end{tabular}

*Significância ao nível de 95\% ( $P<0,05)$; **Significância ao nível de $99 \%(P<0,01)$; e n.s. = não significativo.

Os gráficos de superfície de resposta, obtidos com o modelo descrito na equação 7 , (Figura 2) mostram que o IE das farinhas mistas extrusadas alcança a maior resposta $(3,26)$ em uma formulação de $10 \%$ de FBJ a uma temperatura de extrusão de $130{ }^{\circ} \mathrm{C}$ e a uma rotação do parafuso de $240 \mathrm{rpm}$. Esta variável resposta está relacionada com a capacidade da estrutura do expandido que mantém a integridade das bolhas no material fundido ao sair da matriz do extrusor, sendo que em altas temperaturas ocorre grande redução da viscosidade do produto amiláceo por causa da desintegração do amido propiciando a perda do vapor de água retido na estrutura, ocasionando colapso das bolhas de ar diminuindo o IE [20, 31, 37].

O aumento da rotação do parafuso, que por sua vez está diretamente relacionado com o aumento da energia mecânica específica do sistema, está intimamente relacionado com a

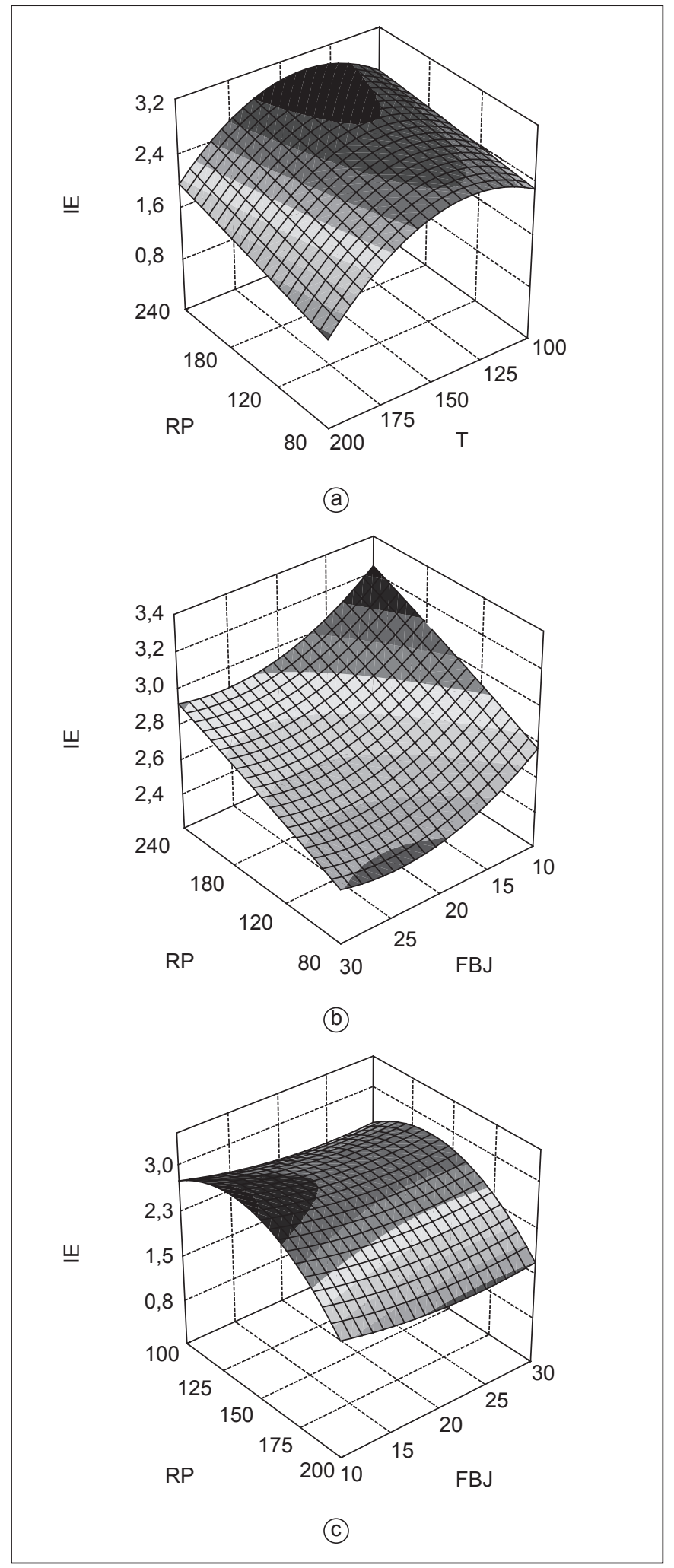

FIGURA 2 - Índice de expansão das farinhas mistas extrusadas de arroz polido e de bagaço de jabuticaba em função de: a) temperatura $x$ rotação do parafuso; $b$ ) temperatura $x$ farinha de bagaço de jabuticaba; e c) rotação do parafuso $x$ farinha de bagaço de jabuticaba.

quebra molecular do amido. Desta forma, o aumento do IE pode estar relacionado com a maior redução do peso mo- 
lecular da matriz amilácea fundida no interior do extrusor o que pode ter contribuído para reduzir a resistência da matriz polimérica à pressão interna das bolhas, que irão favorecer a maior porosidade da estrutura final das farinhas mistas extrusadas. No entanto, alta quebra molecular pela ação da energia mecânica pode, ao contrário, reduzir a expansão final do extrusado [14], que neste caso não foi suficientemente grande para reduzir o IE. A relação inversa entre a porcentagem de farinha de bagaço de jabuticaba e o IE se deve à diminuição da proporção de material amiláceo na mistura e as fibras incluídas não fornecem suficiente material viscoso, afetando as propriedades reológicas do produto extrusado. Outra explicação deste fenômeno pode ser pelo encolhimento do extrusado como reportado por FAN et al. [21]. Recentemente, DELLA VALLE et al. [16] relataram que a temperatura de transição in vitro para a formação das bolhas de ar diminui com a diminuição do conteúdo de amilose. Segundo ILO et al. [30], o arroz contém $15-27 \%$ de amilose, portanto, adicionando-se FBJ nas farinhas é diminuído o teor de amilose, o que, conseqüentemente, favorece o encolhimento dos expandidos e, portanto, diminui o IE.

O ISA está relacionado à quantidade de sólidos solúveis em uma amostra seca, permitindo verificar o grau de severidade do tratamento, em função da degradação, gelatinização, dextrinização e conseqüente solubilização do amido. De acordo com alguns autores [1, 13, 27, 43, 46], o ISA aumenta com a severidade do tratamento, o que também foi constatado por MARTIN-MARTINEZ et al. [37] e MALI \& GROSSMANN [36] em trabalhos com amido modificado, por ZHOU et al. [49] em trabalhos com amido de arroz, por FERNÁNDEZ-GUTIÉRREZ [23] com misturas extrusadas de amido de trigo e caseína, e SACCHETTI et al. [44], trabalhando com extrusados à base de arroz.

Na Tabela 5 e Figura 3, nota-se que a temperatura exerceu um efeito negativo e a rotação do parafuso, um efeito positivo no ISA das farinhas mistas extrusadas. Valores maiores deste índice $(17,55 \%)$ foram em temperaturas próximas de $100{ }^{\circ} \mathrm{C}$, em uma RP de $240 \mathrm{rpm}$ para $10 \%$ de FBJ. O efeito negativo de $\mathrm{T}$ também foi observado por CHANG et al. [15] e MILAN-CARRILLO et al. [40] em expandidos por extrusão de misturas de amido de mandioca e isolados protéicos de soja e em grãos-de-bico, respectivamente.

HASHIMOTO \& GROSSMAN [29], em extrusados de misturas de amido e farelo de mandioca, observaram que o ISA diminui com o aumento do conteúdo de farelo na mistura e com o aumento da temperatura. Estes autores explicaram que as modificações estruturais envolvidas pelas fibras presentes no farelo seriam os responsáveis pela diminuição do ISA. Estas modificações podem ter promovido interações entre a fibra e o amido, reduzindo a solubilidade.

À semelhante conclusão pode-se chegar, neste caso, porém, além das fibras, o teor de proteínas também pode ser responsável pela diminuição do ISA, uma vez que a FBJ contém boa porcentagem de proteína (Tabela 3 ) e esta, em altas temperaturas, favorecem a produção de $-\mathrm{NH}_{2}$ terminal pela desnaturação que, reagindo com os -OH livres origina-

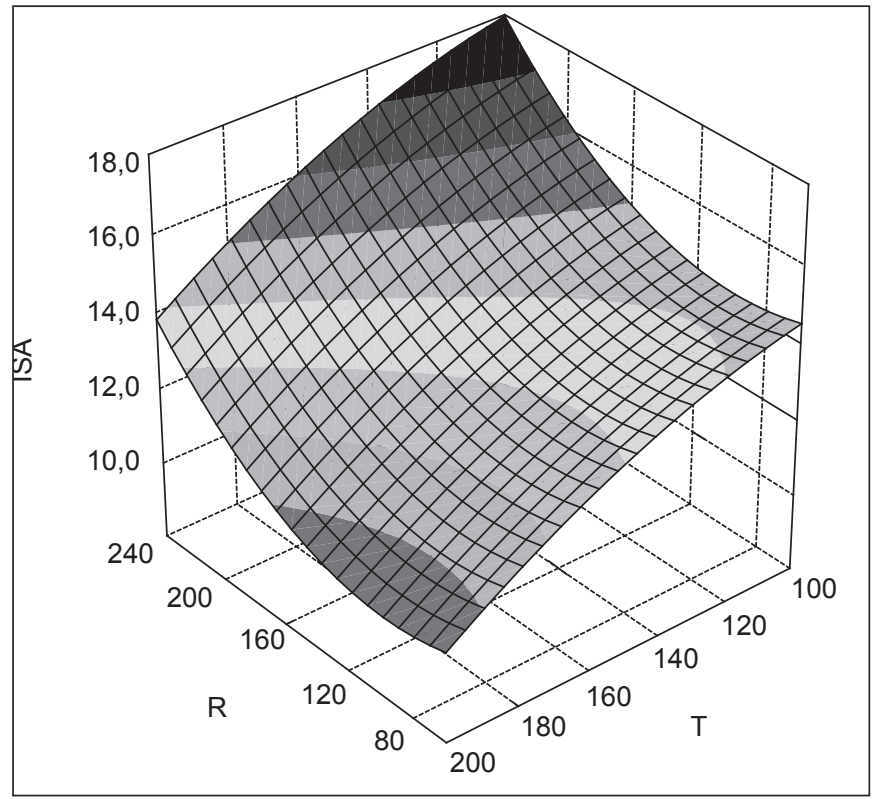

FIGURA 3 - Índice de solubilidade em água das farinhas mistas extrusadas de arroz polido e de bagaço de jabuticaba em função de Temperatura x Rotação do parafuso.

dos pela dextrinização do material amiláceo, propiciaram a formação de complexos não solúveis [22, 24, 28].

\section{4 - CONCLUSÕES}

De acordo com os resultados obtidos e dentro das condições adotadas no presente estudo, conclui-se que: a farinha de bagaço de jabuticaba caracteriza-se como um produto desidratado em pó constituído de partículas poliédricas irregulares de cor roxo-clara, de aroma característico do fruto e de sabor azedo-adstringente e ligeiramente salgado, proporcionando consideráveis quantidades de fibras insolúveis, proteínas e sais minerais.

A expansão dos extrusados aumentou com o aumento da rotação do parafuso e diminuiu com o aumento da adição de farinha de bagaço de jabuticaba, apresentando um comportamento quadrático com o aumento da temperatura. Os expandidos atingiram maior índice de expansão $(3,26 \mathrm{~mm} / \mathrm{mm})$ quando extrusada uma formulação de farinha mista crua de $10 \%$ de bagaço de jabuticaba e $90 \%$ de farinha de arroz polido, para uma temperatura de $130{ }^{\circ} \mathrm{C} \mathrm{e}$ uma velocidade do parafuso de $240 \mathrm{rpm}$.

O índice de solubilidade em água das farinhas mistas extrusadas mostrou que, nos parâmetros do processo de extrusão de $100^{\circ} \mathrm{C}$ e $240 \mathrm{rpm}$, ocorreu maior gelatinização do material amiláceo, tendo como resultado maiores valores deste índice (17,55\%). A FAP misturada com 10\% de FBJ poderá ser usada como matéria-prima para a produção de farinhas pré-gelatinizadas, se as circunstâncias operacionais apropriadas forem aplicadas.

\section{5 - REFERÊNCIAS BIBLIOGRÁFICA}

[1] ALVIM, I. D.; SGARBIERI, V. C.; CHANG, Y. K. Desenvolvimento de farinhas mistas extrusadas à base de fa- 
rinha de milho, derivados de levedura e caseína. Ciênc. Tecnol. Aliment., Campinas, v. 22, n. 2, p. 170-176, maio/ago. 2000.

[2] ANDERSON, R. A. Water absorption and solubility and amylograph characteristics of roll-cooked small grain products. Cereal Chem., Saint Paul, v. 59, n. 4, p. 265-269, Jul./Aug. 1982.

[3] ASCHERI, D. P. R. et al. Efeito da extrusão sobre a adsorção de água de farinhas mistas pré-gelatinizadas de arroz e bagaço de jabuticaba. Ciênc. Tecnol. Aliment., Campinas, v. 26, n. 2, p. 325-335, abr./jun. 2006a

[4] _. Obtenção de farinhas mistas pré-gelatinizadas a partir de arroz e bagaço de jabuticaba: efeito das variáveis de extrusão nas propriedades de pasta. B. CEPPA, Curitiba, v. 24, n. 1, p. 115-144, jan./jun. 2006b

[5] ASQUIERI, E. R. et al. Fabricación de vino blanco y tinto de jabuticaba (Myrciaria jaboticaba Berg) utilizando la pulpa y la cáscara respectivamente. Alimentaria, Madrid, n. 355, p. 97-109, 2004a.

[6] . Vino de jabuticaba (Myrciaria cauliflora Berg): estúdio de las caracterísiticas físico-químicas y sensoriales de los vinos tintos seco y dulce, fabricados con la fruta integral. Alimentaria, Madrid, n. 355, p. 111-121, 2004.

[7] ASSOCIATION OF OFFICIAL AGRICULTURAL CHEMISTS. Official methods of analysis of the association of official agriculture chemists. 17th ed. Washington: AOAC, 2000. 1094 p.

[8] BADUI, D. S. Química de los alimentos, México: Ed. Addison Wesley y Logman de México, 1999.

[9] BOTElHO, L.; CONCEIÇÃO, A.; CARVALHO, V. D. Caracterização de fibras alimentares da casca e cilindro central do abacaxi 'smooth cayenne'. Ciência agrotécnica, Lavras, v. 26, n. 2, p. 362-367, mar./abr. 2002.

[10] BOX, G. E. P.; HUNTER, W. G.; HUNTER, J. S. Statistics for experimenters: an introduction to design, data analysis, and modo building. New York: Wiley and Sons; 1978.

[11] BRASIL. Agência Nacional de Vigilância Sanitária. Regulamento técnico referente à informação nutricional complementar. Portaria n. 27, de 13 de janeiro de 1998. Diário Oficial da República Federativa do Brasil, Brasília, jan. 1998. Disponível em: <http://e-legis.bvs. br/leisref/public/showAct.php?id=97>. Acesso em dec. 2005.

[12] BRUNINI, M. A. et al. Influência de embalagens e temperatura no armazenamento de jabuticabas (Myrciaria jabuticaba (vell) berg) cv 'sabará'. Ciênc. Tecnol. Aliment., Campinas, v. 24, n. 3, p. 378-383, jul./set. 2004

[13] CARVAlHO, R. V.; ASCHERI, J. R. A.; CAL-VIDAL, J. Efeito dos parâmetros de extrusão nas propriedades físicas de pellets $(3 \mathrm{~g})$ de misturas de farinhas de trigo, arroz e banana. Ciência Agrotécnica, Lavras, v. 26, n. 5, p. 1006-1018, set./out. 2002.

[14] CHANG, Y. K. et al. The influence of specific mechanical energy on cornmeal viscosity measured by an on-line system during twin-screw extrusion. Brazilian Journal Of Chemical Engineering, São Paulo, v. 16, n. 3, p. 285-295, set. 1999 .
[15] Twin-screw extrusion of cassava starch and isolated soybean protein blends. Nahrung/Food, Germany, v. 45, n. 4, p. 234-240, Aug. 2001.

[16] Della VAlle, G.; VerGneS, B.; COLONNA, P.; PÁTRIA, A. Relations between rheological properties of molten starches and their expansion behaviour in extrusion. Journal of Food Engineering, Oxford, v. 31, n. 3, p. 277-296, Feb. 1997.

[17] DONADIO, L. C. Jabuticaba [Myrciaria jaboticaba (vell.) Berg]. Jaboticabal: Funep, 2000. 55 p.

[18] DREHER, M.L. Food industry perspective: functional properties and food uses of dietary fiber. In: KRITCHEVSKY, D.; BONFIELD, C. (Edit.) Dietary fiber in health \& disease. Minnesota: Eagan Press, 1995. p. 467-74.

[19] DUARTE, O.; LUDDERS, P.; HUETE, M. Extending storage life of jaboticaba (Myrciaria cauliflora Berg) fruits. In: XIV Congresso Brasileiro de Fruticultura, 1996. Resumos... Curitiba: IAPAR, 1996. v. 1. p. 556.

[20] FAN, J. T.; MITCHELL, J. R.; BLANSHARD, J. M. V. Computer-simulation of the dynamics of bubble-growth and shrinkage during extrudate expansion. Journal of Food Engineering, Oxford, v. 23, n. 3, p. 337-356, Fev. 1994.

[21] ____. The effect of sugars on the extrusion of maize grits: I. The role of the glass transition in determining product density and shape. International Journal of Food Science and Technology, Oxford, v. 31, n. 1, p. 55-65, Jan. 1996.

[22] FERNANDES, M. S. et al. Produtos extrusados expandidos de misturas de canjiquinha e soja para uso como petiscos. Pesquisa Agropecuária Brasileira, Brasília, v. 37, n. 10, p. 1495-1501, out. 2002.

[23] FERNÁNDEZ-GUTIÉRREZ, J. A. et al. Physicochemical properties of casein-starch interaction obtained by extrusion process. Starch/Stärke, Weinheim, v. 56, n. 5, p. 190-198, May. 2004.

[24] FERREIRA, T. A. P. C. Avaliação nutricional do amaranto (Amaranthus caudatus L.) extrusado em diferentes condiçóes de umidade. 1999. $157 \mathrm{f}$. Tese (Doutorado em Pós-Graduação em Ciência dos Alimentos) - Faculdade de Ciências Farmacêuticas, Universidade de São Paulo, São Paulo, 1999.

[25] GOMES, F. P. Curso de Estatística Experimental. 2th ed, São Paulo: ESALQ/USP, 2000.

[26] GUJSKA, E.; KHAN, K. Functional properties of extrudates from high starch fractions of navy and pinto beans and corn meal blended with legume high protein fractions. Journal of Food Science, Chicago, v. 56, n. 2, p. 431-435, Fev. 1991.

[27] GUTKOSKI, L. C. Caracterização tecnológica de frações de moagem de aveia e efeito de umidade e temperatura de extrusão na sua estabilidade. 1997, 241 f. Tese (Doutorado em Pós-Graduação em Tecnologia de Alimentos) - Faculdade de Engenharia de Alimentos, Universidade Estadual de Campinas, Campinas, 1997.

[28] GUY, R. Snack foods. In: GUY, R. (edit.). Extrusion cooking. Boca Raton: Woodhead Publishing Limited, 2001. v. 8, p. 161-181. 
[29] HASHIMOTO, J. M.; GROSSMANN, M. V. E. Effects of extrusion conditions on quality of cassava bran/cassava starch extrudates. International Journal of Food Science and Technology, Oxford, v. 38, n. 5, p. 511-517, Jun. 2003.

[30] ILO, S.; BERGHOFER, E. Kinetics of colour changes during extrusion cooking of maize grits. Journal of Food Engineering, Oxford, v. 39, n. 1, p. 73-80, Jan. 1999.

[31] KÖKSEL, H. et al. Effects of extrusion variables on the properties of waxy hulless barley extrudates. Nahrung/ Food, Germany, v. 48, n. 1, p. 19-24, Feb. 2004.

[32] LAUNAY, B.; KONE, T. Twin-screw extrusion-cooking of corn starch: flour properties of starch pastes. In: ZEUTHEN, P. et al. (edits.). Effects of processing parameters on physical properties on corn starch extrudates expanded using supercritical $\mathrm{CO}_{2}$ injection. Cereal Chemistry, Saint Paul, v. 76, n. 1, p. 63-69, Jan. 1999.

[33] LUE, S.; HSIEH, F.; HU, H. E. Extrusion cooking of corn meal and sugar beet fiber: effects on expansion properties, starch gelatinization and dietary FIber content. Cereal Chemistry, Saint Paul, v. 68, n. 3, p. 227-234, Mar. 1991.

[34] LUSAS, E., RIAZ, M. An introduction extruders and extrusion principles. Extrusion Communiqué, United Kingdom, v. 7, n. 4, p. 9-34, 1994.

[35] MALI, S.; GROSSMANN, M. V. E. Preparation of acetylated distarch adipates by extrusion. Lebensm.-Wiss. u.-Technol., Oxford, v. 34, n. 6, p. 384-389, Sept. 2001.

[36] MAGALHÃES, M. M.; BARROS, R. S.; FINGER, F. L. Changes in structural carbohydrates in developing fruit of Myrciaria jaboticaba. Scentia Horticulturae, Amsterdam, v. 66, n. 1, p 17-22, Sept. 1996.

[37] MARTÍN-MARTÍNEZ, E.S. et al. Starch phosphates produced by extrusion: physical properties and influence on yogurt stability. Starch/Stärke, Weinheim, v. 56, n. 5, p. 199-207, Apr. 2004.

[38] MATTOS, J. R. Frutíferas nativas do Brasil: jaboticabeiras. Porto Alegre: Nobel. 1983.

[39] MELETTI, L. M. M. Propagação de frutíferas tropicais. Guaíba: Livraria e Editora Agropecuária, 2000. p. 145-153.

[40] MILÁN-CARRILLO, J. et al. Optimisation of extrusion process to transform hardened chickpeas (Cicer arietinum, L.) into a useful product. J. Sci. Food Agric., Chichester, v. 82, n. 14, p. 1718-1728, Nov. 2002.

[41] OLIVEIRA, A. L. et al. Caracterização tecnológica de jabuticabas 'sabará' provenientes de diferentes regiões de cultivo. Revista Brasileira de Fruticultura, São Paulo, v. 25, n. 3, p. 397-400, dez. 2003.

[42] PARK, K. H. Elucidation of the extrusion puffing process. 1976. Thesis (Doctor) - University of Illinois, Urbana, 1976.

[43] SACCHETTI, G. et al. Effects of extrusion temperature and feed composition on the functional, physical and sensory properties of chestnut and rice flour-based snack-like products. Food Research International, Oxford, v. 37, n. 5, p. 527-534, Jun. 2004.

[44] SEBIO, L.; CHANG, Y. K. Effects of selected process parameters in extrusion of yam flour (Dioscorea rotundata) on physicochemical properties of the extrudates. Nahrung, Weinheim, v. 44, n. 2, p. 96-101, Mar. 2000.

[45] SOUZA, G. B. et al. Método alternativo para a determinação de fibra em detergente neutro e detergente ácido. São Carlos: Embrapa Pecuária Sudeste, 1999. Boletim de Pesquisa, 4, Fol. 2381.

[46] TOIVONEN, P. M. A.; DEELL, J. R. Chlorophyll fluorescence, fermentation product accumulation, and quality of stored broccoli in modified atmosphere packages ad subsequent air storage. Postharvest Biol. Technol., Amsterdam, v. 23, n. 1, p. 61-69, Jan. 2001.

[47] WHALEN, P. J. et al. Measurements of extrusion effects by viscosity profile using the rapid viscoanalyser. Cereal Foods World, Saint Paul, v. 42, n. 6, p. 469-475, Jun. 1977.

[48] WILBANK, M. V.; CHALFUN, N. N. J.; ANDERSEN, O. O. The jaboticaba in Brazil. Proceedings of the Americans Society for Hoticutural Science, Alexandria, v. 27 A, p. 57-69, 1983.

[49] YATES, A. A.; SCHLICKER, A.; SUITOR, C. W. Dietary reference intakes: the new basis for recommendations for calcium and related nutrients, B vitamins, and choline. J. Am. Diet. Assoc., United States, v. 98, n. 6 , p. 699-706, Jun. 1998.

[50] ZHOU, Z.; ROBARDS, K.; HELLIWELL, S.; BLANCHARD, C. Composition and functional properties of rice. International Journal of Food Science and Technology, Oxford, v. 37, n. 8, p. 849-868, Dec. 2002.

\section{6 - AGRADECIMENTOS}

Os autores agradecem ao Centro de Tecnologia de Alimentos da Empresa Brasileira de Pesquisa Agropecuária do Rio de Janeiro (CTAA/EMBRAPA - RJ) pelo suporte técnico. À Fazenda Jabuticabal do Município de Nova Fátima do Estado de Goiás pela matéria-prima fornecida. 